\title{
Archéologie du futur. Une rétro-modélisation de l'architecture expérimentale de Pascal Häusermann, Claude Costy et Jean-Louis Chanéac
}

\author{
Archaeologie of the future. \\ Retro modeling from experimental architecture of the late \\ sixties (Häusermann, Costy, Chanéac)
}

\author{
Mélina Ramondenc ${ }^{*}$ et Philippe Marin \\ Univ. Grenoble Alpes, ENSAG, School of Architecture, MHA, 38000 Grenoble, France
}

\begin{abstract}
Résumé. Cet article présente une expérimentation pédagogique de rétromodélisation de projets d'architecture expérimentale des années soixante. L'exercice s'appuie sur le matériau d'une recherche doctorale en histoire de l'architecture en revisitant les projets de son corpus au prisme des outils et des cultures numériques. L'analyse de ce corpus, composé des travaux des architectes Jean-Louis Chanéac, Pascal Häusermann et Claude Costy, membres du Groupe International d'Architecture Prospective (GIAP), nous permet de ré-investiguer des formes architecturales qui anticipaient sur leurs moyens de production et de conception, et de procéder ainsi d'une forme d' " archéologie du futur » (Jameson, 2005).
\end{abstract}

Mots-clés. Architecture expérimentale, modélisation géométrique, pensée algorithmique, construction robotisée.

\begin{abstract}
This article presents a pedagogical experiment in retromodelling experimental architecture projects from the 1960s. The exercise is based on the material of a $\mathrm{PhD}$ research in architectural history and it allows projects to be revisited from the perspective of contemporary digital techniques and cultures. The analysis of the research corpus, namely the work of architects Jean-Louis Chanéac, Pascal Häusermann and Claude Costy, members of the Groupe International d'Architecture Prospective (GIAP), allows us to re-investigate architectural forms that anticipated their means of production and design, thus proceeding from a form of "archaeologie of the future" (Jameson, 2005)
\end{abstract}

Keywords. Experimental architecture, complex geometric modelling, algorithmic thinking, robotic construction.

ªmondenc.m@grenoble.archi.fr - philippe.marin@grenoble.archi.fr 


\section{Introduction}

Alors que s'ouvre l'ère post industrielle (Touraine 1970), nombreux sont les architectes qui prennent la mesure du retard accumulé dans le secteur du logement, dont la production massive s'organise toujours selon des principes édictés avantguerre. Autour de Michel Ragon, critique autodidacte, se structure un réseau de chercheurs qui veulent inventer le «futur de l'architecture» (Hollein 1965): le Groupe International d'Architecture Prospective (GIAP). L'objectif de ce groupe hétéroclite est de regrouper des concepteurs de tous les pays pour produire et promouvoir des solutions architecturales et urbaines qui répondent aux bouleversements sociaux et sociétaux annoncés, et surtout de réaliser. Jean-Louis Chanéac, Pascal Häusermann et Claude Costy adhèrent à ce programme de recherche et d'action et rejoignent les fondateurs du GIAP.

La dimension prospective des travaux de ces trois architectes crée parfois un anachronisme entre leurs projets et les moyens alors à leur disposition pour les réaliser. Ce constat est à la base d'une proposition pédagogique visant à reconsidérer ces audacieux témoignages d'une époque où l'architecture cherchait à anticiper l'évolution de ses moyens de production. Le séminaire de rétromodélisation proposé sonde cette production architecturale en utilisant les outils numériques. Procédant d'une forme d'« archéologie du futur » (Jameson 2005) pour reprendre les termes de l'historien américain Fredric Jameson, l'examen de ces projets prospectifs permet de mettre à jour des logiques de projet et de production de l'architecture particulièrement en phase avec les questionnements contemporains. Alors que l'impression numérique des bâtiments devient réalité, les moyens technologiques dont nous disposons aujourd'hui semblent avoir rattrapé les rêves de ces architectes souvent qualifiés de visionnaires.

Cet article est organisé en deux parties. La première présente le contexte historique des projets que nous étudions et les thématiques soulevées par la prospective architecturale à la fin des années soixante. La seconde présente plus précisément deux de ces projets d'architecture et les intérêts pédagogiques de leur rétromodélisation.

\section{Du Groupe International d'Architecture Prospective à l'association Habitat Evolutif, la recherche d'une personnalisation de l'habitat.}

\subsection{Une architecture prête-à-habiter}

Dès la fin des années soixante, "l'innovation technique, par les jeux de trames qu'elle autorise, est pensée comme levier du renouveau architectural qui rompt avec le chemin de grue. »[1] Dans leur recherche d'alternatives aux tours et aux

\footnotetext{
${ }^{1}$ «PAN - Programme Architecture Nouvelle (1972-1987) », publié le 6 novembre 2015 sur http://www.urbanisme-puca.gouv.fr [dernière consultation le 3/04/2020]
} 
barres, les architectes se saisissent des possibilités offertes par les logiques de l'industrialisation ouverte et de l'essor des matériaux issus de la pétrochimie pour concevoir à moindre coût des éléments combinables et modulables. Henri Van Lier explique : «l'édifice contemporain n'est plus une pièce unique, c'est un produit industriel. Ce qui ouvre deux voies. Ou bien l'on étudie avec tout le soin désirable un prototype d'édifice multipliable en un nombre indéfini d'exemplaires [...] avec les inconvénients de monotonie et d'inadaptation que l'on sait. Ou bien l'on essaye de créer de vrais éléments, c'est-à-dire des matériaux qui, par leur chimie, leur physique, leur forme sont produits aux moindres frais, avec le maximum de garanties de résistance, et qui en même temps se prêtent à des combinaisons très riches, permettant de tisser des ensembles architecturaux infiniment variés » (Van Lier 1959). Cette deuxième voie est celle qu'explorent ensemble Chanéac, Häusermann et Costy. Ils s'emploient ainsi à répondre au besoin de personnalisation (Baudrillard 1965) de cette architecture de masse. Cela passe par la possibilité de choisir entre différents modèles proposés, d'y ajouter des options, et de décider de leur combinaison. Pour ajouter encore aux éventualités, Chanéac appelle de ses vœux une « architecture industrialisée poétisée » mise en œuvre par des " ouvriers poètes, chargés de créer des altérations à l'uniformité du système " (Chanéac 2005). Les premières recherches de Chanéac et du couple HäusermannCosty sont publiées en 1963 dans le même numéro de la revue l'Architecture d'Aujourd'hui intitulé Recherches, et qui présente notamment les travaux de Yona Friedmann, Paul Maymont, et Nicolas Schöffer, membres fondateurs du GIAP. Après avoir pris connaissance de leurs recherches respectives, Chanéac, Costy et Häusermann décident de travailler ensemble au sein du groupe Habitat Evolutif. Principalement portée par Pascal Häusermann, l'association permet la coopération étroite des trois architectes rejoints par Antti Lovag. Häusermann travaille alors au projet des Domobiles.

\subsection{Une architecture sur-mesure}

Parallèlement à leur recherche d'une architecture prête-à-habiter, Chanéac, Häusermann et Costy expérimentent une architecture sur-mesure. Si ces deux démarches paraissent à priori contradictoires, elles sont cimentées par le même idéal : personnaliser l'habitat. Enthousiasmés par leurs découvertes respectives au Polytechnique de Londres et aux Etats-Unis auprès d'élèves de Frank Lloyd Wright, Häusermann et Costy s'intéressent à la technique du gunitage, qui consiste à projeter au pistolet le béton liquide treillis métallique recouvert d'un support d'enduit. Cette mise en œuvre du béton armé leur semble optimale au regard des propriétés conjuguées des matériaux : fer et béton. Fort de cette intuition partagée, le couple expérimente une architecture guidée par la recherche d'une vérité constructive et d'une économie de moyens. Dès son retour de Londres en 1958, Pascal Häusermann réalise une coque de béton autoportée couvrant une surface de $70 \mathrm{~m}^{2}$ pour un total de 5000 francs de l'époque. La grande liberté qu'autorise cette technique permet à Pascal Häusermann et Claude Costy de construire des édifices aux formes singulières. De 1966 à 1970, ils travaillent ainsi à de nombreux projets d'habitations individuelles, et inventent une architecture dimensionnée par les 
usages et les désirs de ses habitants. La propre maison du couple, construite en 1968, est l'exemple le plus marquant de cette architecture buissonnière, esquissée le soir pour être réalisée le lendemain. Au début des années soixante-dix, Chanéac expérimente aussi cette technique pour deux projets majeurs : un magasin de meubles (Ain) tout en courbes puis sa propre maison (Savoie). Son approche de la technique est toutefois assez différente de celle du couple Häusermann-Costy : la mise en œuvre de ses projets est totalement maîtrisée et ne laisse aucune place à l'improvisation.

\section{Exercices pédagogiques de rétro-modélisation}

\subsection{Présentation des cas d'études rétro-modélisés}

Le séminaire pédagogique a été l'occasion de modéliser cinq projets : la Ville cratère (Chanéac, 1963-1969), le Balcon de Belledonne (Häusermann et Costy, 1966), la Maison Coquillage (Häusermann et Costy, 1967), les Domobiles (Häusermann, 1970-1972), le centre-ville de Douvaine (Chanéac, Costy, et Häusermann, 1971-1978). Tout l'enjeu de ce panel était de rendre compte du large spectre d'outils de conception de ces architectes, du projet le plus standardisé (les Domobiles), au moins standardisé (le Balcon de Belledonne). En outre, cette sélection est représentative des différentes échelles abordées par ces concepteurs, du module habitable au projet de territoire. Le corpus d'analyse sur lequel s'est basé le séminaire pédagogique comportait des textes, des documents iconographiques et les bâtiments, pour établir la rétro-modélisation la plus informée possible. Dans cet article, nous présentons deux projets : Les Domobiles (1970-1974) réalisées par Pascal Häusermann associé à Patrick Le Merdy, décorateur d'intérieur, et le Balcon de Belledonne (1966) signé de Pascal Häusermann et de son associée et épouse Claude Costy.

Ces deux cas d'étude révèlent des démarches opposées et soulèvent des enjeux pédagogiques spécifiques. L'architecture du Balcon de Belledonne s'accompagne d'une géométrique complexe, les coques sont faites de surfaces à doubles courbures, leurs génératrices sont des courbes nurbs (acronyme de l'expression anglaise « Non-Uniform Rational Basis Splines ») complexes, l'épaississement des surfaces génère des torsions, le calcul des intersections de solides est délicat. $\mathrm{Ce}$ travail de modélisation impose la maîtrise des concepts géométriques numériques. Les Domobiles se constituent également de coques, réalisées à partir de sphères ou d'ellipsoïdes, mais les principes qui sous-tendent ce projet reposent plus particulièrement sur des questions de combinatoire et d'assemblages de modules. Les deux approches établissent d'étroites relations avec l'architecture dite non standard (Migayrou 2003). Les géométries sont complexes, elles sont réalisées à partir de courbes et leurs modélisations sont rendues possibles par l'usage des formalismes mathématiques appelées spline [2] et nurbs. L'histoire du

\footnotetext{
${ }^{2}$ Le terme spline se traduit par le nom français cerce. Une cerce est un outil de dessin, ou de construction, permettant de tracer des courbes continues. Le terme est transposé dans les outils numériques pour qualifier les fonctionnalités de réalisation des objets « courbes ».
} 
développement de ces instrumentations a été récemment retracée par Alastair Townsend (Townsend 2014). Par ailleurs ces projets questionnent les relations à la fabrication et à la construction. L'un s'appuie sur des logiques d'auto-construction, l'autre renvoie à des principes de préfabrication et d'industrialisation. Les deux projets révèlent des spatialités renouvelées, la délimitation entre sol, mur et plafond s'efface au profit de surfaces continues et organiques.

\subsubsection{Les Domobiles (1970-1974)}

En 1971, Haüsermann s'associe à l'architecte d'intérieur Patrick Le Merdy pour développer les Domobiles. Ce projet d'architecture combinatoire est basé sur l'assemblage de différents types de cellules fonctionnelles entièrement industrialisées en coques de polyester, qui intègrent mobiliers et équipements. Leur combinaison doit permettre de générer des habitations individuelles modulables, en grande quantité et à faible coût, qui soient toutes uniques. Pour plus de variété, le système permet d'associer les cellules Domobiles à des modules d'autres concepteurs par le biais d'un connecteur commun. Vouées à être commercialisées en grandes surfaces ou par correspondance, puis assemblées librement par leur propriétaires, ces cellules esquissent une architecture de consommation. Lauréat du deuxième Programme Architecture Nouvelle (PAN) en 1972, le projet est soutenu par la caisse des Dépôts, permettant la réalisation d'un prototype.

Les Domobiles proposent «une architecture neutre, disponible, mobile, par conséquent une architecture par l'habitant plutôt que par l'architecte, et où ce dernier jouerait un rôle de conseiller, de cadre, mais surtout s'appliquerait à concevoir les supports, les joints, les étanchéités assurant au plus bas prix la liberté de chacun » (Van Lier 1959). Le projet des Domobiles permet de saisir comment l'exploration de l'industrialisation des matières plastiques permet de tendre vers un design intégral : les concepteurs passent d'une pensée en trame à une pensée en moule, se libèrent de l'orthogonalité pour penser en négatif et de volumes habités.

\subsubsection{Le Balcon de Belledonne (1966)}

Au début des années soixante, Pascal Häusermann et Claude Costy se lancent dans la construction de coques en béton projeté. En 1966, Daniel Telmont fait appel au couple pour réaliser Le Balcon de Belledonne, à Sainte-Marie-du-Mont (Isère) : un complexe de loisirs ouvert uniquement l'été. L'ensemble architectural s'installe dans la pente comme un organisme vivant : chaque élément du programme génère sa propre enveloppe. La coque principale offre un vaste espace libre qui contient la salle de restaurant et offre une vue panoramique sur le massif de Belledonne. Elle s'ouvre sur une terrasse en belvédère, soutenue par un système de poteaux évoquant un réseau racinaire. Depuis cette terrasse, une passerelle mène à la piscine et au sauna.

\subsection{Rétro-modélisation et usage des technologies numériques}


Si les projets étudiés questionnent à l'origine les techniques constructives ainsi que leurs modalités de production, ils restent a priori éloignés des technologies numériques. Il nous paraît cependant intéressant de relire ces expérimentations au prisme des techniques contemporaines de modélisation. Ainsi ce travail de rétromodélisation présente un double intérêt. Il est d'abord le moyen d'obtenir une connaissance fine des projets étudiés. La modélisation numérique de ces architectures implique un redimensionnement précis, elle impose à travers la réalisation du modèle en trois dimensions une résolution géométrique et parfois une interprétation des dessins originaux, initialement réalisés en deux dimensions, et présentant parfois des incohérences géométriques. Ces résultats peuvent alors alimenter un travail d'analyse et de caractérisation de ces architectures. Il est ensuite un prétexte pédagogique pour exercer les savoirs et savoir-faire de nos étudiants en matière de culture numérique. Nous avons retenu trois thèmes spécifiques qui font l'objet d'un approfondissement.

\subsubsection{Pensée combinatoire et modélisation paramétrique}

Un premier exemple porte sur les questions combinatoires mises en œuvre dans le projet des Domobiles. Les différentes unités peuvent s'assembler à la demande en fonction des besoins des futurs habitants et des spécificités du lieu dans lequel elles s'installent. Cette personnalisation de la composition de l'habitation renvoie à des principes aujourd'hui familiers, mais ils sont conçus à un moment de l'histoire de l'architecture numérique qui voit naître, d'une part les techniques de génération appelées grammaire de forme (Stiny et Gips 1972) et d'une part les premières expérimentations informatiques d'assistance des habitants dans la configuration de leurs habitations. En Amérique du Nord les travaux de l'Architecture Machine Group de Nicholas Negroponte au Massachusetts Institute of Technology (MIT) (Negroponte 1975) explorent l'usage des ordinateurs pour accompagner les habitants dans le choix et la configuration de leurs habitats. Les travaux Yona Friedman (Friedman 1971) conduits dans cette même institution prennent place dans cette problématique d'implication de l'habitant dans la définition de son habitat alors que l'architecte joue le rôle du concepteur d'un système architectural combinable.

Nous envisageons un configurateur de Domobiles dans un environnement de modélisation paramétrique. Après une modélisation géométrique de chacune des cellules de base (Figure 1), deux «clusters» (Figure 2) ont été implantés pour permettre la configuration d'un «complexe» Domobiles. Le premier cluster, dénommé module, intègre la définition de la géométrie d'une unité (chambre, salon ou module de liaison), ainsi que le choix des faces d'entrée et de sortie. Le second cluster, nommé assemblage, permet de combiner deux modules et de les relier par un plan d'assemblage (Figure 3). 


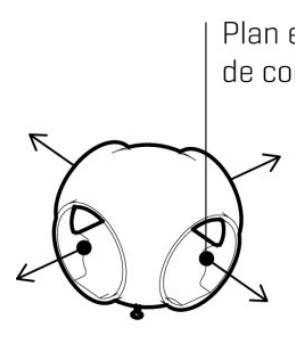

Module de liaison

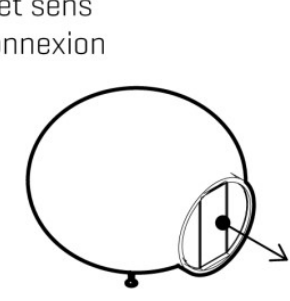

Module chambre

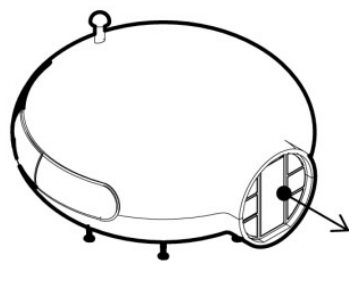

Module Salon

Figure 1. Modélisation des modules

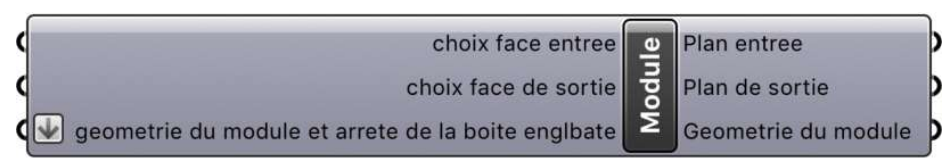

Paramètre du module

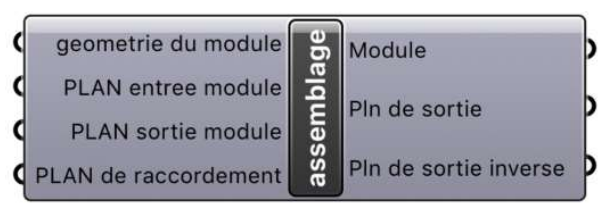

Assemblage des modules

Figure 2. Vue des clusters
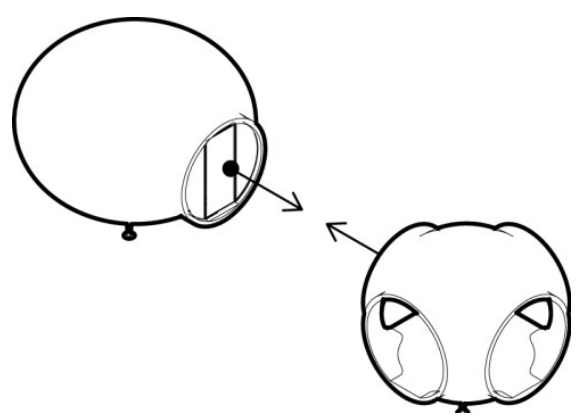

Figure 3. Plan d'assemblage de deux modules

La programmation visuelle de grasshopper offre une interface de configuration des Domobiles en reliant successivement les clusters assemblages. La Figure 4 montre le modèle paramétrique issu de grasshopper. Celui-ci est composé de 8 modules assemblés suivant différentes directions. La Figure 5 représente la configuration correspondante. 


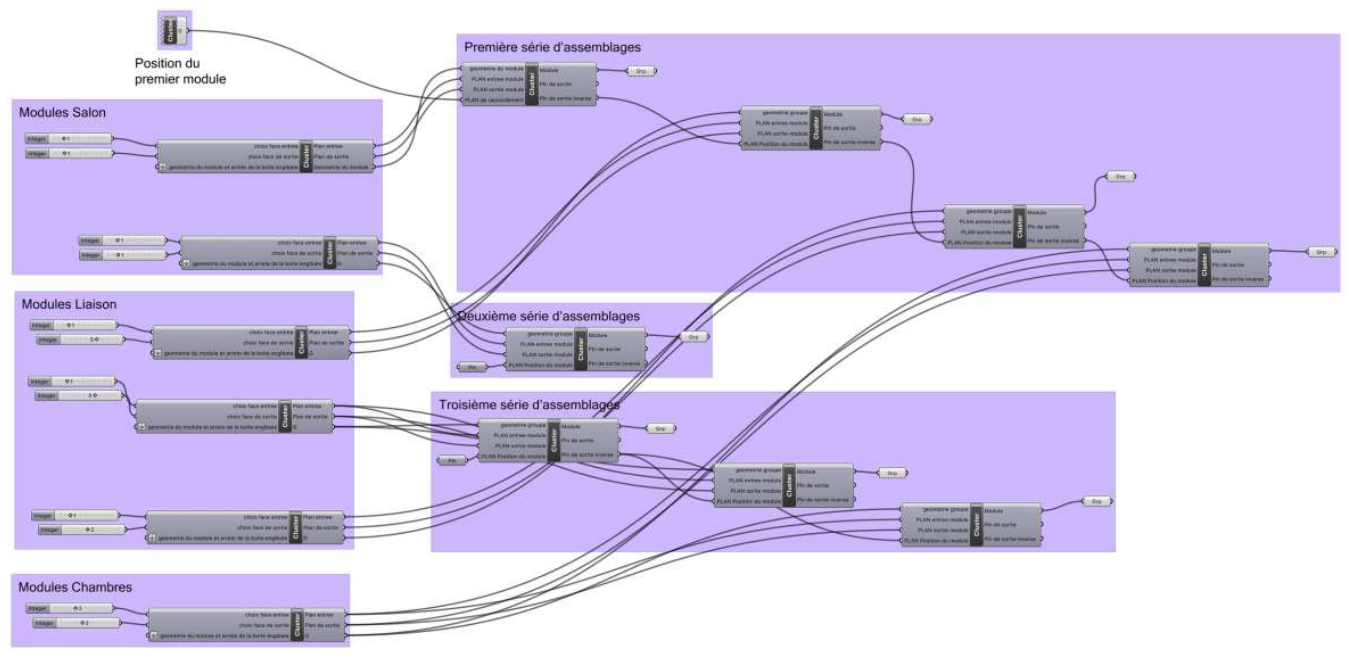

Figure 4. Vue du modèle grasshopper pour une configuration de 8 modules

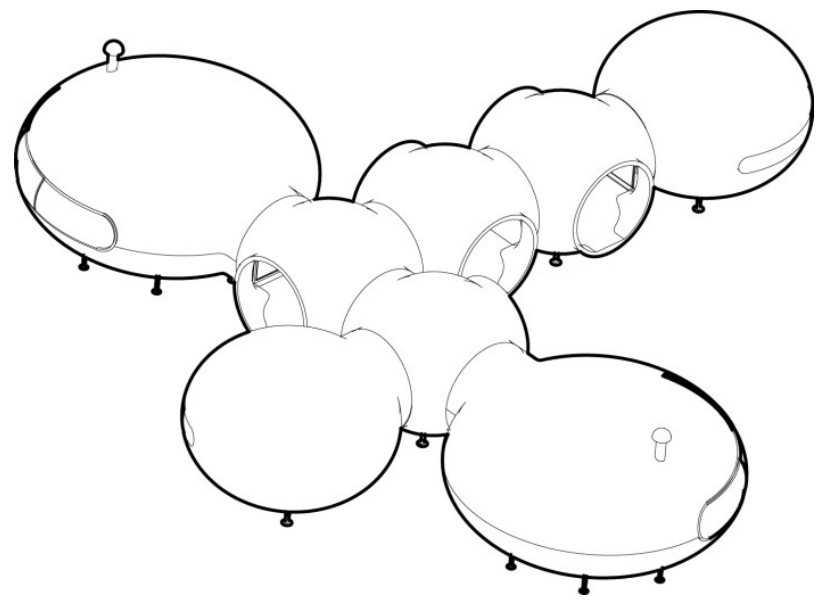

Figure 5. Possible configuration de 8 modules

L'intérêt de grasshopper repose sur sa dimension interactive. Le branchement des clusters génère en temps réel la représentation géométrique correspondante. Il est également possible de reconfigurer la composition en modifiant l'identifiant des faces de connexion depuis les paramètres d'entrées des clusters modules. La principale limite actuelle de notre proposition repose sur l'impossibilité de générer une configuration à partir de la spécification paramétrique du nombre de modules souhaités. Le nombre de modules n'est pas un paramètre de notre script; pour constituer un ensemble habité il est nécessaire de positionner sur la scène de programmation autant de «clusters modules» que nécessaire. Le processus de configuration n'est pas paramétrique, il reste linéaire et nécessite l'assemblage successif des clusters. Ainsi, si l'hypothèse du développement d'un outil d'assistance à la configuration de son habitat, à destination des usagers, devait être 
prolongée, il apparaitrait nécessaire d'envisager une interface plus accessible pour des utilisateurs non-experts.

\subsubsection{Modélisation géométrique complexe}

L'étude des architectures expérimentales présente un second intérêt à travers la complexité formelle de certains de ces projets. Ainsi le Balcon de Belledonne révèle des complexités géométriques que seuls les logiciels contemporains de modélisation sont capables d'approcher. Coque, surface à doubles courbure, congés et raccord de surfaces, surfaces par balayage, surface de décalage, sont quelquesunes des fonctions géométriques mises en œuvre pour assurer la modélisation de ces édifices. L'illustration suivante montre une modélisation du projet Balcon de Belledonne (Figure 6) Une série de surfaces à double courbure est modélisée, les surfaces initiales sont décalées pour former une coque puis découpées pour créer des ouvertures. Les surfaces sont reliées par des congés pour assurer des continuités. La modélisation en trois dimensions donne lieu à la génération de nouveaux dessins en deux dimensions et permet par ailleurs la production de maquettes imprimées en trois dimensions. La maquette composée de plusieurs pièces est assemblée et offre un support de médiation du projet. Sa spatialité, son organisation, son installation dans la pente sont explicitées à l'aide de ces modèles physiques et numériques en trois dimensions. La Figure 7 montre la maquette physique réalisée à l'aide de la découpe laser des éléments constitutifs du terrain et de l'impression 3D par frittage des modules d'habitation.
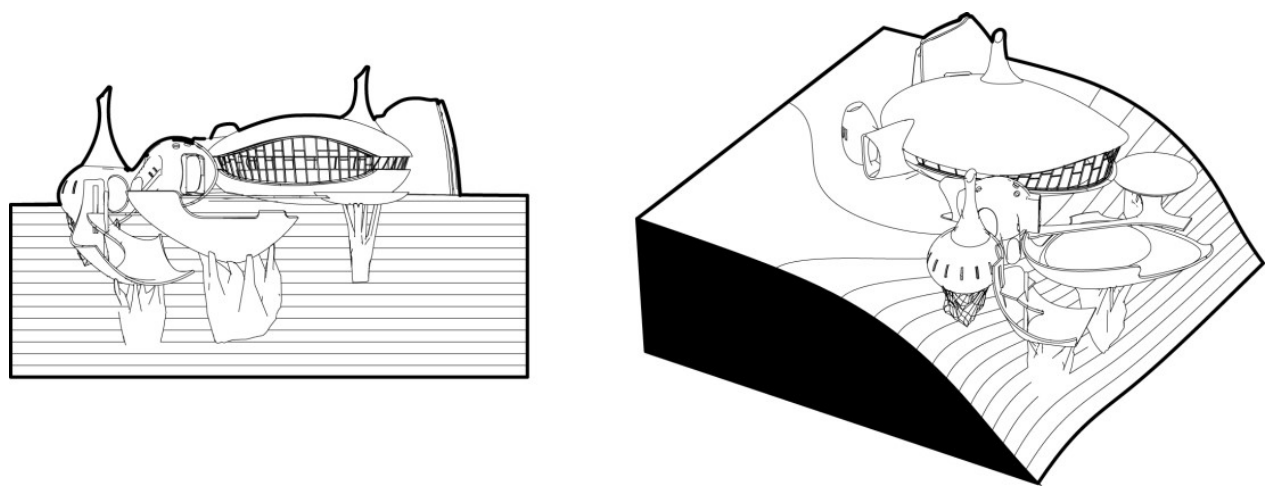

Figure 6. Élévation et axonométrie du projet Balcon de Belledonne 


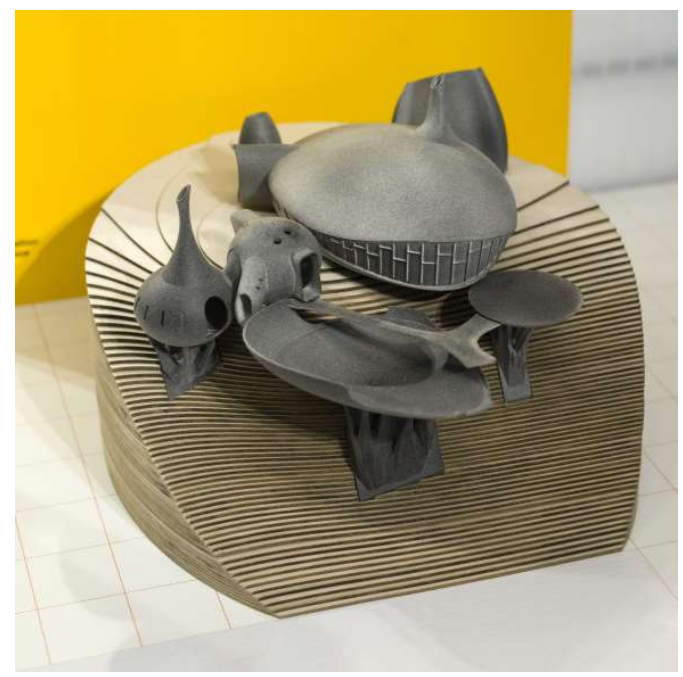

Figure 7. Maquette du Balcon de Belledonne, impression 3D (C) CAUE de Haute-Savoie, Anthony Denizard

La rétro-modélisation de ces projets exerce, chez les étudiants, leurs capacités de modélisation géométrique, elle nécessite un savoir-faire relatif aux formes courbes et complexes, elle demande une bonne appréhension des questions d'échelle et de dimensionnement dans la perspective de fabrication et de maquettage à l'aide des techniques d'impression en trois dimensions.

\subsubsection{Hypothèses constructives et fabrication robotisée}

La troisième thématique abordée à travers le travail de rétro-modélisation de ces architectures expérimentales porte sur des questions constructives et interroge les processus de réalisation de ces projets. Une réinterprétation des solutions constructives mises en œuvre initialement est opérée en transposant ces méthodes dans un contexte enrichi de techniques numériques. Initialement, la construction du projet Balcon de Belledonne passe par la mise en forme d'un grillage soutenue par des tiges métalliques courbées à l'aide de gabarits. Cette structure accueille ensuite une projection de béton, réalisée successivement en couches minces. Les techniques de fabrication additive et robotisée permettent de revisiter ces processus. Une première hypothèse porte sur une mise en forme d'un treillis structurel sur site et à l'aide d'un bras robotisé (Hack et al. 2017). Une deuxième hypothèse envisage l'impression 3D du treillis à l'aide d'un dépôt de fil chaud (Hack 2018). Ces techniques font l'objet d'expérimentations et certaines solutions sont commercialisées par des sociétés telles que AI-Build ${ }^{3}$ ou Branch Technology ${ }^{4}$. La projection du béton sur ce support (Figure 8) peut alors être envisagée et réalisée à l'aide d'une tête d'extrusion portée par un bras ou un portique.

\footnotetext{
${ }^{3}$ http://www.ai-build.com Consulté le 15 décembre 2019

4 https://www.branch.technology Consulté le 15 décembre 2019
} 


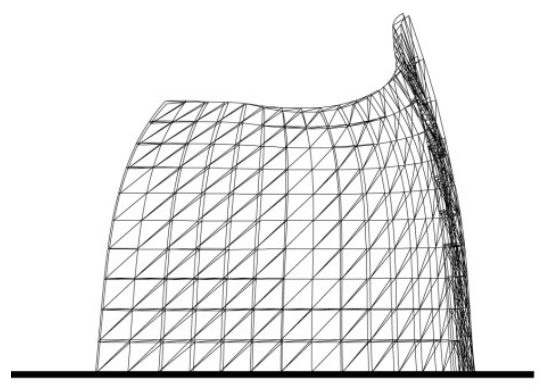

Vue de face

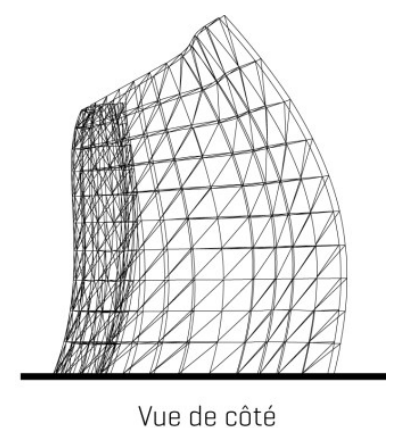

Vue de côté

Figure 8. Exemple de structure en treillis d'une des coques

\section{Conclusion}

Nous avons décrit un exercice pédagogique visant l'acquisition de compétences, de savoirs et de savoir-faire en matière de culture numérique. L'originalité de notre proposition repose sur le fait que l'exercice prend appui sur une recherche de doctorat. La rétro-modélisation est composée à partir de documents originaux et d'une connaissance des concepts et des idées. L'exercice nous permet de mobiliser trois compétences particulières : la première porte sur les logiques combinatoires et une pensée algorithmique, la seconde sur des questions de modélisation de géométries complexes et la troisième sur une transposition des méthodes constructives avec l'aide des techniques contemporaines de fabrication robotisée et additives. L'ancrage historique des projets étudiés nous permet de renforcer la culture architecturale de nos étudiants tout en envisageant les modalités contemporaines d'instrumentation de la conception et de la construction. Des relations sont construites entre les postures initiales de ces architectes et les perspectives ouvertes par les cultures numériques et les techniques contemporaines : auto-construction et approche participative, personnalisation de l'habitat et standardisation, industrialisation et automatisation de la construction, par exemple. Dans le futur, nous envisageons d'élargir l'exercice en sondant d'autres travaux des membres du GIAP.

Les travaux ont été réalisés sous la coordination de Philippe Marin et avec la participation de l'équipe pédagogique composée de Tchely Shin et Jason Sachs. Ils ont été soutenus par une allocation de la Structure Fédérative de Recherche Territoires en réseaux (UGA). Les maquettes physiques réalisées dans le cadre de ce séminaire ont été intégrées à l'exposition «Conquêtes spatiales » produite par le CAUE de Haute-Savoie en mars 2020.

\section{Bibliographie}

Baudrillard, Jean (1968). Le système des objets, Paris : Gallimard [réed.2016], 288 pp. Chanéac, Jean-Louis (2005). Architecture interdite, Paris : Editions du Linteau, 213 pp. Friedman, Yona (1971). Pour l'architecture scientifique, Paris : Pierre Belfond.

Hack, Norman Peter, (2018). « Mesh Mould: A Robotically Fabricated Structural Stay-inPlace Formwork System $»$ PhD Thesis, Zurich: ETH Zurich. https://doi.org/10.3929/ethz-b-000263345. 
Hack, Norman Peter, Timothy Wangler, Jaime Mata-Falcón, Kathrin Dörfler, Nitish Kumar, Alexander Walzer, Konrad Graser, et al. (2017). «Mesh mould: an on site, robotically fabricated, function formwork ».

Hollein, Hans, (1965) «Zukunft der Architektur », Revue Bau, Vienne.

Jameson, Fredric (2005). Archeologies of the Future, The Desire Called Utopia and Other Science Fictions, London : Verso, 431 p.

Migayrou, Frédéric (2003). Architectures non standard, Catalogue d'exposition (10 déc. 2003-1er mars 2004) Paris : Editions du Centre Pompidou, 223 p.

Negroponte, Nicholas (1975). Soft architecture machines, Cambridge : The MIT Press.

Ragon, Michel, Où vivrons-nous demain ? (1963). Paris : Robert Laffont, 214 pp.

Stiny, George, et James Gips (1972). «Shape Grammars and the Generative Specification of Painting and Sculpture ». In Information Processing 71, Amsterdam, pp.1460-1465.

Touraine, Alain (1970). «La société post-industrielle », Population, 25e année, n³, pp. 684-685.

Townsend, Alastair (2014). " On the Spline: A Brief History of the Computational Curve », International journal of interior architecture + spatial design Issue 3

Van Lier, Henri (1959). Les Arts de l'Espace, Tournai : Casterman. 